NA LI ${ }^{1}$

TEH Y. TAN ${ }^{1, \infty}$

U. GÖSELE ${ }^{2}$

\title{
Transition region width of nanowire hetero- and pn-junctions grown using vapor-liquid-solid processes
}

\author{
${ }^{1}$ Department of Mechanical Engineering and Materials Science, Duke University, \\ Durham, NC 27708, USA \\ 2 Max-Planck Institute of Microstructure Physics, Weinberg 2, 06120 Halle, Germany
}

\section{Received: 19 November 2007/Accepted: 10 December 2007 \\ Published online: 12 January 2008 • () Springer-Verlag 2008}

ABSTRACT The transition region width of nanowire heterojunctions and pn-junctions grown using vapor-liquid-solid (VLS) processes has been modeled. With two constituents or dopants I and II, the achievable width or abruptness of the junctions is attributed to the residual I atom/molecule stored in the liquid droplet at the onset of introducing II to grow the junction, and the stored I atom/molecule consumption into the subsequently grown crystal layers. The model yields satisfactory quantitative fits to a set of available Si-Ge junction data. Moreover, the model provides a satisfactory explanation to the relative junction width or abruptness differences between elemental and compound semiconductor junction cases, as well as a guideline for achieving the most desirable pn-junction widths.

PACS 81.07.-b; 64.75.Jk; 61.46.Km

\section{Introduction}

Semiconductor nanowires (NW) have been regarded as having great potentials in future electronic, optoelectronic and thermoelectric applications [1-3]. Thus, extensive studies have been performed on the preparation, property, and growth kinetics of nanowires and nanowire structures with either hetero- or pn-junctions [1-9]. Nanowire heterojunction structures of various semiconductors have been fabricated, e.g., Si-Ge [3-5], GaAs-InAs [6] and GaP-GaAs $[7,8]$. The performance of these devices depends closely upon the width or abruptness of the junction region. Among several possible device configurations, the axial heterojunction and pn-junction structures are obviously advantageous, because they can be directly formed during VLS nanowire growth by a switching of the source material; hence they do not require additional junction fabrication processes. However, instead of possessing the ideal abruptness on an atomic scale, NW heterojunctions grown via the VLS method always have graded composition transition regions with a relatively large junction width $[4,5,7]$, i.e., a sizable fraction of the nanowire diameter. We suggest that this phenomenon is a characteristic feature of the VLS growth method.

The VLS nanowire growth process consists of three steps. First, the vapor phase precursor diffuses to and dissociates at the liquid droplet surface. Second, the semiconductor atoms/molecules diffuse through the droplet to reach the liquid-solid interface. Third and finally, the semiconductor atoms/molecules adapt the solid crystal lattice structure, and thus the wire grows. In experiments of growing the heterojunctions, the supply of the vapor phase source of material I, which is the semiconductor material of the already grown nanowire section, e.g., $\mathrm{Si}$, can in principle be abruptly decreased or terminated, while simultaneously the supply of the vapor phase source of semiconductor material II, e.g., Ge, abruptly activated. This abrupt change of the source material supply, however, does not warrant the growth of abrupt junctions. At the onset of junction growth, i.e., at the beginning instant of growing the II or II-containing nanowire layers on an already grown I nanowire section, there exist a definite number of residual I atoms left in the liquid droplet, which is concomitant with the prior growth of the I-only section. After the junction starts to grow, these residual I atoms are removed via the consumption into the newly grown nanowire layers. This is a gradual process instead of an abrupt one because, as determined by thermodynamics, as soon as some I atoms are consumed the liquid is replenished by II atoms flowing in from the vapor phase, and I and II atoms will start to mix and rapidly become thoroughly mixed. This decreases the concentration of the still left I atoms in the liquid so that less I atoms will be consumed out of the liquid in a subsequently grown nanowire layer. Therefore, during junction growth, the number of I atoms in the liquid droplet decreases only gradually, and so is the number of I atoms incorporated into each new solid layer. We believe that this process is the very basic reason why the experimentally grown heterojunctions all possess graded transition regions with large junction widths, instead of abrupt ones with atomic scale junction widths. Although in theory it is impossible to grow atomic scale abrupt heterojunctions, it has been reported that some III-V compound semiconductor heterojunctions can be fairly sharp $[1,8,9]$, while much wider junctions were always obtained for the $\mathrm{Si}-\mathrm{Ge}$ 
cases. In a recent experiment, it has also been found that the width of the Si-Ge heterojunctions is directly proportional to the nanowire size [5].

Based on the above prognosis of the VLS process, we have formulated a model to analyze the nanowire heterojunctions. Numerically calculated results based on this model fit very well with the experimentally measured data [5]. The model also successfully explained several related growth features. The same model is also applicable in analyzing concentration profiles of pn-junctions formed by the VLS process.

\section{$2 \quad$ Formulation of the model}

To formulate the model, we adopt the following assumptions: (1) the dissolution of semiconductor atoms from the vapor source into the liquid is very fast and the liquid surface always maintains equilibrium with the vapor phase; the diffusion/mixing of these atoms in the liquid is so rapid that the liquid has a uniform composition; (2) the nanowire growth rate $v$ is the same at all times; (3) the liquid droplet is regarded as a pseudo-binary alloy, with the metal (M) being the solvent, while semiconductor I and II atoms behave as two isotopes of the same solute material. Assumption (3) leads to the consequence that the solid-liquid segregation coefficients of I and II are the same, which in turn leads to

criterion (a): that, at any instant $t$ the molar ratio of I and II in the liquid droplet is the same as that in the nanowire layer grown at $t$ within the time duration $\mathrm{d} t$.

For convenience, we will also assume that the number of semiconductor atoms in the liquid droplet is a constant. Under this assumption, at the onset of junction growth, i.e., at $t=0$, let the strength of the I vapor source be suddenly decreased from $1(100 \%)$ to $1-\alpha$, with $0<\alpha \leq 1$ holding, and the vapor source II of the strength $\alpha$ be suddenly activated. Now a nanowire layer of an alloy consisting of I and II will start to grow, while in the liquid the total number of semiconductor atoms is maintained at the same constant value of $n_{\mathrm{o}}=n_{\mathrm{Io}}$ at all times. Denote the number of atoms of I and II in the liquid by $n_{\mathrm{I}}^{1}$ and $n_{\mathrm{II}}^{1}$, respectively, and those in the solid nanowire by $n_{\mathrm{I}}^{\mathrm{s}}$ and $n_{\mathrm{II}}^{\mathrm{s}}$, respectively. The model is formulated by considering the flow rates of I and II atoms into and out of the liquid droplet, respectively denoted as $\dot{n}_{\mathrm{I}}^{1}$ (in), $\dot{n}_{\text {II }}^{1}$ (in), $\dot{n}_{\mathrm{I}}^{1}$ (out), and $\dot{n}_{\mathrm{II}}^{1}$ (out); see Fig. 1 . In this manner, in steady state the rate of $(\mathrm{I}+\mathrm{II})$ atoms flowing into the liquid droplet from the vapor phase is constant at all times and exactly balances that flowing out of the liquid droplet to grow the wire. That is, relations $\dot{n}_{\mathrm{I}}^{1}(\mathrm{in})+$ $\dot{n}_{\mathrm{II}}^{1}($ in $)=c, \dot{n}_{\mathrm{I}}^{1}$ (out) $+\dot{n}_{\mathrm{II}}^{1}$ (out) $=-c$, and $\dot{n}_{\mathrm{I}}^{1}$ (in) $+\dot{n}_{\mathrm{II}}^{1}($ in $)+\dot{n}_{\mathrm{I}}^{1}$ (out) $+\dot{n}_{\mathrm{II}}^{1}$ (out) $=0$ hold. Here $c$ is a constant.

The I atoms flowing out of the liquid droplet are consumed into the growing nanowire layer. Hence, $\dot{n}_{\mathrm{I}}^{1}($ out $)=-\dot{n}_{\mathrm{I}}^{\mathrm{s}}$ holds, where $\dot{n}_{\mathrm{I}}^{\mathrm{s}}$ is the rate of incorporating I atoms into the solid nanowire layer at time $t$. Thus, $\dot{n}_{\mathrm{I}}^{\mathrm{s}}=c_{\mathrm{I}}^{\mathrm{s}}\left(v \pi r_{\mathrm{s}}^{2} / \Omega\right)$, where $c_{\mathrm{I}}^{\mathrm{s}}$ is the fractional concentration of $\mathrm{I}$ in the as grown solid nanowire layer, $\left(v \pi r_{\mathrm{s}}^{2} / \Omega\right)$ is the rate of incorporating $(\mathrm{I}+\mathrm{II})$ atoms into the layer, with $v$ being the nanowire growth rate, $r_{\mathrm{s}}$ the nanowire radius, and $\Omega$ the volume of an average atom in the nanowire (which will also be later used as the average atom volume in the liquid, since the two volumes are approximately equal). Clearly, then,

$\dot{n}_{\mathrm{I}}^{1}($ out $)=-c_{\mathrm{I}}^{\mathrm{s}}\left(v \frac{\pi r_{\mathrm{s}}^{2}}{\Omega}\right)$

holds. Now, criterion (a) yields $n_{\mathrm{I}}^{\mathrm{s}} / n_{\mathrm{II}}^{\mathrm{s}}=$ $n_{\mathrm{I}}^{1} / n_{\mathrm{II}}^{1}$, from which we obtain

$c_{\mathrm{I}}^{\mathrm{s}}=c_{\mathrm{I}}^{\mathrm{IR}}$,

\section{vapor phase}

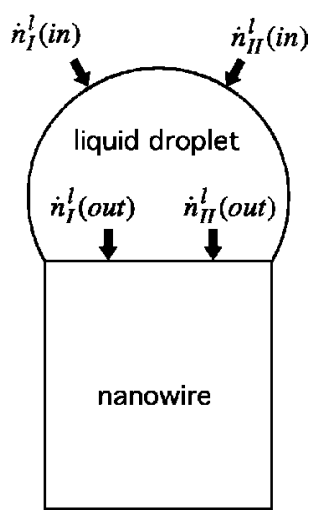

FIGURE 1 Schematic of the VLS process during the junction growth with the flow rates indicated where $c_{\mathrm{I}}^{\mathrm{s}}=n_{\mathrm{I}}^{\mathrm{s}} /\left(n_{\mathrm{I}}^{\mathrm{s}}+n_{\mathrm{II}}^{\mathrm{s}}\right)=n_{\mathrm{I}}^{\mathrm{s}} / n_{\mathrm{Io}}^{\mathrm{s}}$ is the fractional I concentration in the nanowire, while $c_{\mathrm{I}}^{\mathrm{lR}}=n_{\mathrm{I}}^{1} /\left(n_{\mathrm{I}}^{1}+n_{\mathrm{II}}^{1}\right)=$ $n_{\mathrm{I}}^{1} / n_{\text {Io }}^{1}$ is the reduced fractional I concentration in the liquid. Note that $c_{\mathrm{I} / \mathrm{II}}^{\mathrm{IR}}$ is defined only for I and II, and does not involve M. From (1) and (2), we obtain

$\dot{n}_{\mathrm{I}}^{1}($ out $)=-c_{\mathrm{I}}^{\mathrm{R}}\left(v \frac{\pi r_{\mathrm{s}}^{2}}{\Omega}\right)=-\frac{n_{\mathrm{I}}^{1}}{n_{\mathrm{Io}}^{1}} v \frac{\pi r_{\mathrm{s}}^{2}}{\Omega}$.

Using the relations $\dot{n}_{\mathrm{I}}^{1}$ (in) $+\dot{n}_{\mathrm{I}}^{1}$ (out) $=$ 0 and $n_{\mathrm{I}}^{1}=n_{\mathrm{Io}}^{1}$, which hold prior to and at the onset of the heterojunction growth process, i.e., for $t \leq 0$, we obtain $\dot{n}_{\mathrm{Io}}^{1}$ (in) $=v \pi r_{\mathrm{s}}^{2} / \Omega$. After the onset of junction growth, the I vapor source flow rate is assumed to have dropped to $(1-\alpha) \dot{n}_{\mathrm{Io}}^{1}$ (in), where $0<\alpha \leq 1$. Thus, for $t>0$,

$\dot{n}_{\mathrm{I}}^{1}($ in $)=(1-\alpha) \dot{n}_{\mathrm{Io}}^{1}$ (in $)=(1-\alpha) v \frac{\pi r_{\mathrm{s}}^{2}}{\Omega}$

Inside the liquid droplet, the change rate of $n_{\mathrm{I}}^{1}$ is $\mathrm{d} n_{\mathrm{I}}^{1} / \mathrm{d} t=\dot{n}_{\mathrm{I}}^{1}($ in $)+\dot{n}_{\mathrm{I}}^{1}(\mathrm{out})$, i.e.,

$\frac{\mathrm{d} n_{\mathrm{I}}^{1}}{\mathrm{~d} t}=(1-\alpha) v \frac{\pi r_{\mathrm{s}}^{2}}{\Omega}-\frac{n_{\mathrm{I}}^{1}}{n_{\mathrm{Io}}^{1}} v \frac{\pi r_{\mathrm{s}}^{2}}{\Omega}$,

where $\alpha=0$ and $n_{\mathrm{I}}^{1}=n_{\mathrm{Io}}^{1}$ for $t<0$, and $0<\alpha \leq 1$ for $t \geq 0$. Apply (5) to heterojunction growth for $t \geq 0$ with the initial condition being $c_{\mathrm{I}}^{1}=1$ at $t=0$. The solution is

$$
\begin{aligned}
c_{\mathrm{I}}^{\mathrm{IR}}=\frac{n_{\mathrm{I}}^{1}}{n_{\mathrm{Io}}^{1}}= & (1-\alpha) \\
& +\alpha \exp \left(-\frac{v}{n_{1 o}^{1}} \frac{\pi r_{\mathrm{s}}^{2}}{\Omega} t\right) .
\end{aligned}
$$

Analogously, one obtains

$$
\begin{aligned}
c_{\mathrm{II}}^{\mathrm{IR}}= & \frac{n_{\mathrm{II}}^{1}}{n_{\mathrm{II} o}^{1}}=1-c_{\mathrm{I}}^{\mathrm{lR}} \\
& =\alpha\left[1-\exp \left(-\frac{v}{n_{1 o}} \frac{\pi r_{\mathrm{s}}^{2}}{\Omega} t\right)\right] .
\end{aligned}
$$

Let $n_{\mathrm{Io}}=\beta \pi r_{1}^{3} C_{\mathrm{o}} / 3$, where $\beta$ is the size factor of the droplet specified as a fraction of a sphere, $C_{0}$ is the concentration of $(\mathrm{I}+\mathrm{II})$ atoms in the liquid, $r_{1}$ is the liquid droplet radius, and $\Omega \approx$ $1 / C_{\mathrm{oo}}$, with $C_{\mathrm{oo}}$ being the total volumetric concentration of $(\mathrm{I}+\mathrm{II}+\mathrm{M})$ atoms 
in the liquid, we obtain, via $c_{\mathrm{I}}^{\mathrm{s}}=c_{\mathrm{I}}^{\mathrm{IR}}$ and $c_{\mathrm{II}}^{\mathrm{s}}=c_{\mathrm{II}}^{\mathrm{IR}}$,

$c_{\mathrm{I}}^{\mathrm{s}}=(1-\alpha)+\alpha \exp \left(-\frac{3}{\beta \gamma^{3} c_{\mathrm{o}}} \frac{v t}{r_{\mathrm{s}}}\right)$,

$c_{\mathrm{II}}^{\mathrm{s}}=\alpha\left[1-\exp \left(-\frac{3}{\beta \gamma^{3} c_{\mathrm{o}}} \frac{v t}{r_{\mathrm{s}}}\right)\right]$.

Here $c_{\mathrm{o}}=C_{\mathrm{o}} / C_{\mathrm{oo}}$ is the fractional semiconductor (I+ II) atom concentration in the liquid droplet, $\gamma$ is the ratio of the diameters of the liquid droplet and the nanowire, i.e., $\gamma=r_{1} / r_{\mathrm{s}}$, and $\beta$ is between 2 to 4 . For nanowires with very large diameters, the liquid droplet is nearly a hemisphere with $\beta$ approaching the lower limit 2, while for those with very small diameters the liquid droplet is nearly a sphere and $\beta$ approaches the upper limit 4.

In our model, $\alpha$ also denotes the maximum fractional concentration of II in the solid wire, i.e., $c_{\mathrm{II} \text { max }}^{\mathrm{s}}=\alpha$. Define $\eta=\frac{c_{\mathrm{II}}^{\mathrm{s}^{\prime}}}{\alpha}$, with $c_{\mathrm{II}}^{\mathrm{s}^{\prime}}$ being a given constant value of $c_{\mathrm{II}}^{\mathrm{s}}$. For nanowire heterojunctions grown in the same experiment but with different wire diameters, we obtain

$\frac{L_{\mathrm{j}}}{r_{\mathrm{s}}}=-\frac{\ln (1-\eta)}{\frac{3}{\beta \gamma^{3} c_{\mathrm{o}}}}$,

where $L_{\mathrm{j}}=v t$, which is the length of the nanowire grown since the onset of the heterojunction growth process. The abruptness of a junction is defined as the value of $L_{\mathrm{j}}$ for a given $\eta$. Equation (10) indicates that $L_{\mathrm{j}}$ is directly proportional to $r_{\mathrm{s}}$.

\section{A quantitative application: fit of a set of experimental results}

In this section we use (8)(10) to fit a set of experimentally measured $\mathrm{Si}-\mathrm{Ge}$ heterojunction data [5]. First, (8) and (9) are used to fit an experimentally measured concentration profile of a Si-Ge junction, and second, (10) is used to fit a set of junction abruptness data, all due to Clark et al. [5]. The calculated and experimental results of the Si-Ge concentration profile are shown together in Fig. 2, wherein it is seen that the fit is excellent. The used parameters are listed in Table 1 . The values of $\beta$ and $\gamma$ cannot be measured from experiments, and were thus used as fitting parameters. In the experiment

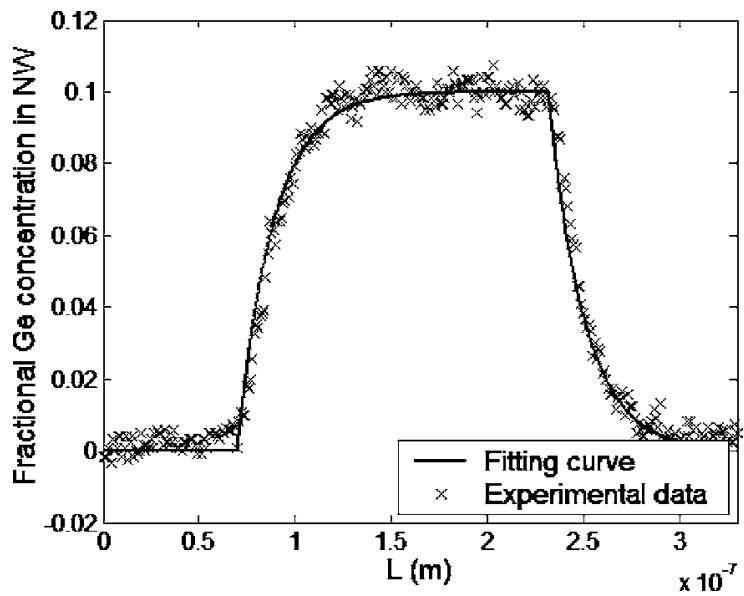

FIGURE 2 Fitting of the experimentally measured concentration profile in the transition region of a Si-Ge heterojunction [5] using the present model

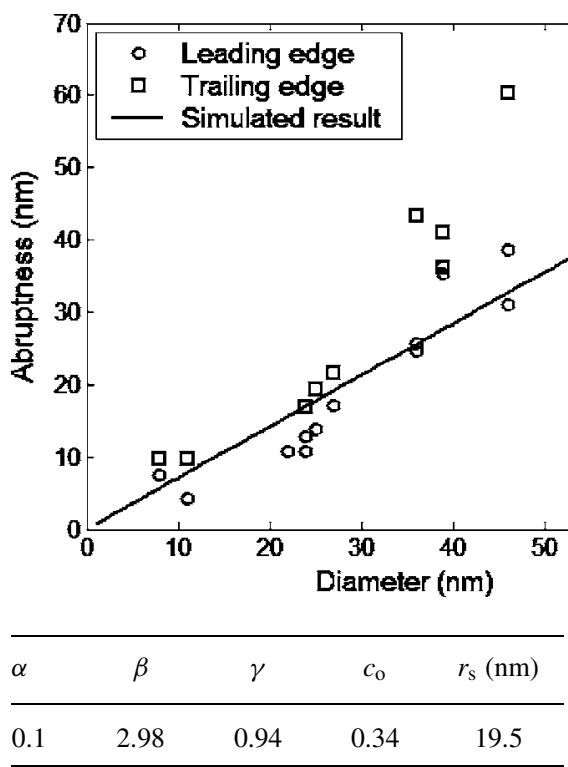

TABLE 1 Parameter values used in obtaining the fitting curve in Fig. 2

of Clark et al. [5], one factor differed slightly from that the model was based on. The Si source strength was held as constant at the onset of Ge source (with the strength $\alpha=0.1$ ) instead of being reduced accordingly to maintain the total vapor source flow strength. Therefore the Si vapor source still had a normalized strength 1 , and the total vapor strength was 1.1. However, as long as $\alpha$ is small which was satisfied in the experiment, (8) and (9) can closely approximate the results.

From the experimental profiles such as the one shown in Fig. 2, Clark et al. [5] have measured the junction abruptness, defined according to the following criteria: (1) for the leading edge of the $c_{\mathrm{II}}^{\mathrm{s}}(\mathrm{Ge})$ profile, it is the additional nanowire length grown since the onset of II until $c_{\mathrm{II}}^{\mathrm{s}}$ reaches $90 \%$ of its maximum value $(\eta=0.9)$; and (2) for the trailing edge of the $c_{\mathrm{II}}^{\mathrm{s}}(\mathrm{Ge})$ profile, it is the additional nanowire length grown since the offset of II until $c_{\text {II }}^{\mathrm{s}}$ decreased to $10 \%$ of its maximum value $(1-\eta=0.1)$. They concluded that the $\mathrm{Si}-\mathrm{Ge}$ junction abruptness is directly proportional to the nanowire radius $r_{\mathrm{s}}$. This liner dependence of the junction abruptness on $r_{\mathrm{s}}$ is consistent with the predicted of our (10), which shows that $L_{\mathrm{j}} \propto r_{\mathrm{s}}$. Using the same parameters for obtaining the fitting results shown in Fig. 2, we calculated that $L_{\mathrm{j}} / r_{\mathrm{s}}=1.43$ for their experimental results. This is depicted by the straight fitting-line in Fig. 3. It is seen that the fitting is satisfactory.

\section{$4 \quad$ Qualitative applications}

It appears that the data of Clark et al. [5] is up to now the only available set of experimental results suitable for quantitative application of the present model. The present model is not applicable to the only other set of quantitative $\mathrm{Si}-\mathrm{Ge}$ heterojunction 
data, that of Zakharov et al. [4], obtained using the molecular beam epitaxy (MBE) method. This is because in the MBE case the nanowire growth rate is controlled by surface diffusion $[4,10]$, while in the present model reaction at the liquid-solid interface is assumed to control the nanowire growth. Nonetheless, we can use the present model to qualitatively explain two kinds of experimental results.

\subsection{The relative abruptness of III- $V$ and $\mathrm{Si}-\mathrm{Ge}$ heterojunctions}

First, our (10) also relates the junction abruptness to the semiconductor solubility in the liquid droplet. The smaller the solubility is, the sharper the junction becomes, as demonstrated by the simulation results in Fig. 4. Here for simplicity we assumed that the droplet is a hemisphere and hence $\alpha=1$. Although quantitative data are not available, it is known that the transition regions of some III-V heterojunctions $[1,8,9]$ can be much sharper than those of Si-Ge $[4,5]$. This is the case because the solubilities of the group $\mathrm{V}$ elements are small in $\mathrm{Au}$, while the solubilities of the group IV elements $\mathrm{Si}$ and Ge are both over 20 at. \%. Thus, the $\mathrm{Si}-\mathrm{Ge}$ structure junction width is comparable to the wire diameter $[4,5]$, while those of the III-V compounds [1,8.9] are much sharper.

\section{2 \\ Applying the model to nanowire pn-junctions}

In addition to semiconductor heterojunctions, pn-junctions can be formed during nanowire growth via the VLS method by programming the dopant sources in the vapor phase. The fundamental physical mechanisms governing the junction widths are the same as those for the formation of heterojunctions. Therefore, the model holds also for the case of pn-junctions, and can be applied to analyze the dopant/carrier concentration profiles of these junctions. The formulation for the pn-junction transition region is similar to that of the heterojunction case, only simpler. First, at $t=0$, it may be assumed that dopant $\mathrm{dI}$ inflow flux terminates and dopant dII inflow flux starts to act, where the prefix d specifies dopant. Second, since the dopant concentration is but a very small fraction of the total number of atoms in the liquid droplet $n_{\mathrm{oo}}$, it is a constant at all times irrespective of adjustment in the dopant dII inflow flux strength, which shall maximally not exceed, e.g., $10^{-4}$ of $n_{\text {oo }}$. We further assume that the segregation coefficient between the solid nanowire and liquid droplet is 1 for each dopant. Follow the same procedure as formulating the heterojunction case, we obtain

$$
\begin{aligned}
& c_{\mathrm{dI}}^{\mathrm{s}}=\exp \left(-\frac{3}{\beta \gamma^{3} c_{\mathrm{o}}} \frac{v t}{r_{\mathrm{s}}}\right), \\
& c_{\mathrm{dII}}^{\mathrm{s}}=\alpha\left[1-\exp \left(-\frac{3}{\beta \gamma^{3} c_{\mathrm{o}}} \frac{v t}{r_{\mathrm{s}}}\right)\right],
\end{aligned}
$$

For the case of pn-junction, the factor $\alpha$ is the ratio of the source strengths between dII and dI, i.e., the source strength of dII divided by that of dI, which can be either smaller or larger than 1.

We can calculate the dopant/carrier concentration profiles to illustrate the relation between junction widths and

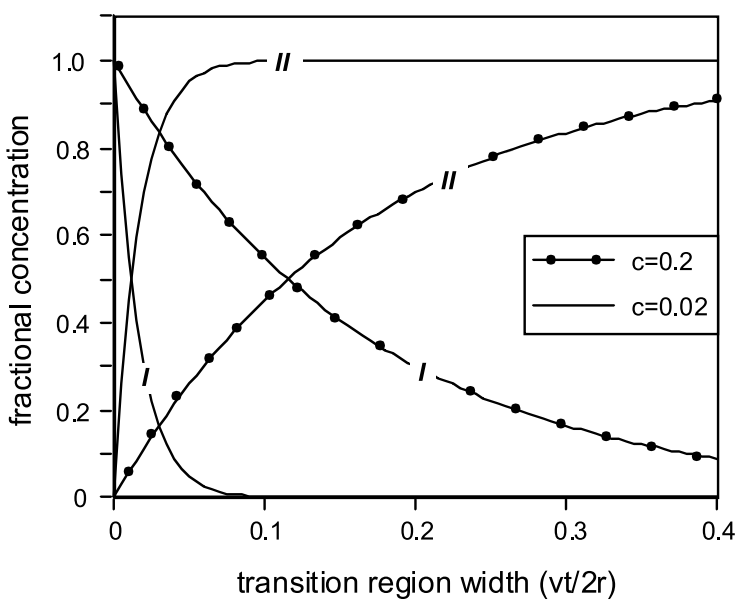

FIGURE 4 The simulated semiconductor heterojunction abruptness, displayed in concentration profiles of dopants I and II, for cases with different semiconductor solubilities in the Au liquid. For III-V compound case the solubility of the group $\mathrm{V}$ elements in $\mathrm{Au}$ is small, taken as 0.02 , resulting in the junction being more abrupt. For Si-Ge, the solubilities of both $\mathrm{Si}$ and $\mathrm{Ge}$ in $\mathrm{Au}$ are fairly large, $\sim 0.2$, resulting in the junction being much less abrupt dopant vapor source strengths using (11) and (12). While it seems impossible to totally or even just substantially eliminate the large graded transition region of the hetero- or pn-junctions, it is nevertheless possible to shrink the region width by a fairly large range in the pn-junction case by simply using stronger dII source materials. To illustrated this point, we show in Figs. 5 and 6 the calculated pn-junction transition region dopant concentrations for several different strengths of dII. It is seen that, the pn-junction width narrows as the dII strength increases, irrespective of whether the dII source is stronger (Fig. 5) or weaker (Fig. 6) than the dI source. Since quantitative concentration profiles of pn-junction are presently not available, it is desirable to obtain such experimental results to check the validity of the present model.

\section{$5 \quad$ Discussions}

In this paper we have proposed a model to explain the width of the VLS grown heterojunctions which are characterized by graded transition regions far from being abrupt on a monolayer scale. Our model attributes this graded nature of these junctions to the residual I atoms stored in the liquid droplet and their mixing with the subsequently introduced II atoms after the onset of junction growth. These factors render the incorporation of II atoms into and the elimination of I atoms from the liquid droplet as gradual processes that cannot produce sharp junction widths on a monolayer scale.

The fact that at the onset of the growth process, residual I atoms exist in the liquid droplet is a natural consequence of growing the I nanowire section. In order to grow an atomically abrupt junction these residual I atoms must be eliminated at once, and the II atoms simultaneously and instantaneously introduced into the liquid. As a consequence of thermodynamics, it appears that there is no way to strictly or even just substantially achieve this purpose. To start with, growth of the I nanowire section relies upon the presence of supersaturated I atoms in the liquid droplet up to the onset of growing the II or II-containing layers. Termination of the I layer growth is achieved by terminating the appropriate vapor 


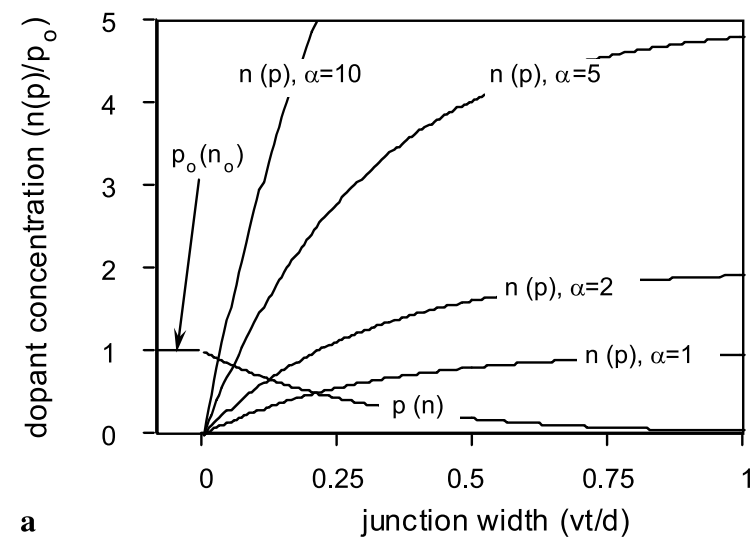

FIGURE 5 Normalized concentration profiles for pn-junctions under different second dopant (II) strengths, all of which are larger than the first dopant (I) strength: (a) The dopant concentration profiles, and (b) The carrier concentration profiles

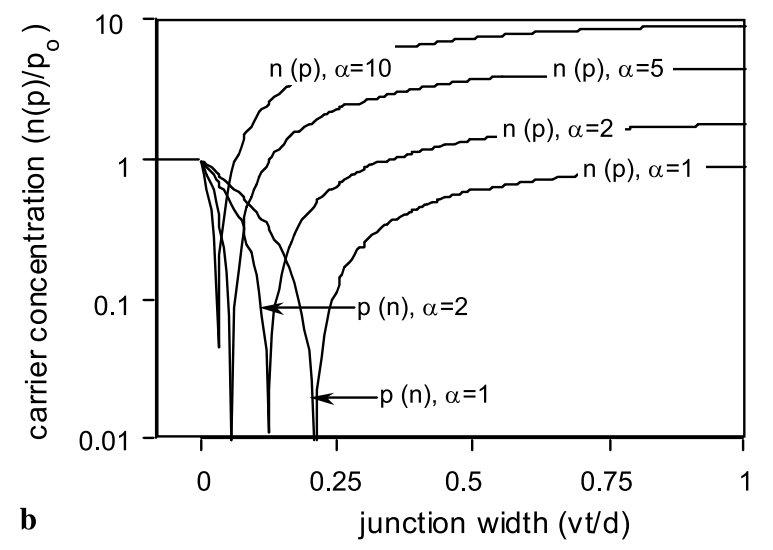

phase source material, which only stops the further supply of I atoms to the liquid droplet but does not and cannot remove the I atoms already in it. These stored residual atoms will be eventually eliminated, by two mechanisms, the consumption mechanism and the evaporation mechanism. The consumption mechanism acts to remove these atoms by consuming them into the further grown solid nanowire layers, and the evaporation mechanism acts by evaporating them back into the ambient. Our present model is based on the consumption mechanism, with the potential contribution from the evaporation mechanism ignored. Judging from the satisfactory fits obtained between our model and the relevant experimental results, our approach to the problem seems to be justified. This can be true, because there are good physical reasons why the evaporation mechanism should not be as important. First, up to now all nanowire heterojunctions that are of interests are grown using semiconductors with very low thermal equilibrium vapor pressures, and hence it is doubtful in any experiment that the contribution of the evaporation mechanism can be prominent. Second, even if it is, then at some point in time, dissolution of the I atoms from the already grown nanowire back into the liquid droplet will take place to replenish I atoms in the liquid, which occurs when the ambient vapor phase I source pressure drops below that can maintain the I nanowire in the stable state when the wire neither grows nor shrinks. These arguments are true for both heterojunctions and pn-junctions whose formulation follows the same model but with slight differences.

In experiments the assumption of the constant semiconductor atom number becomes invalid if the strength of II becomes larger than that of I. Under such conditions, the nanowire growth rate $v$, its diameter $2 r_{\mathrm{s}}$, the liquid droplet diameter $2 r_{1}$, and the total semiconductor concentration $c$ in the liquid can be all changing with time with the increased II semiconductor source material strength. A general description of such a case can still not be obtained. This situation does not arise for the pn-junction case, as the dopant concentration is always much smaller than the semiconductor atom concentration. For the heterojunction case, we can nonetheless extend our model to a slightly less general but still very common case. This is the case for which the wire grows at a constant diameter but both $v$ and $r_{1}$ change with semiconductor concentration $c$ in the liquid droplet (or the total semiconductor atom number $n$ for a given droplet with a constant $\mathrm{Au}$ atom number). In this section, we will discuss the methodology for the formulation of this case. We still use the assumptions (1) and (3). Here let I be turned off at the onset of II. The formulation of the cases for which I was not completely turned off will be similar.

Now $v, n$ and $c$ are all time-dependent variables with initial values being $v_{0}, n_{0}$ and $c_{0}$, respectively. The flow rate equations are

$\dot{n}_{\mathrm{I}}^{1}($ in $)=0$,

$\dot{n}_{\mathrm{I}}^{1}$ (out) $=-\frac{n_{1}}{n} v \frac{\pi r_{\mathrm{s}}^{2}}{\Omega}$,

$\dot{n}_{\mathrm{II}}^{1}($ in $)=\alpha v_{0} \frac{\pi r_{\mathrm{s}}^{2}}{\Omega}$, and

$\dot{n}_{\mathrm{II}}^{1}($ out $)=-\frac{n_{2}}{n} v \frac{\pi r_{\mathrm{s}}^{2}}{\Omega}$.

Therefore we obtain

$$
\begin{aligned}
& \frac{\mathrm{d} n_{\mathrm{I}}^{1}}{\mathrm{~d} t}=-\frac{n_{1}}{n} v \frac{\pi r_{\mathrm{s}}^{2}}{\Omega}, \\
& \frac{\mathrm{d} n_{\mathrm{II}}^{1}}{\mathrm{~d} t}=\alpha v_{0} \frac{\pi r_{\mathrm{s}}^{2}}{\Omega}-\frac{n_{2}}{n} v \frac{\pi r_{\mathrm{s}}^{2}}{\Omega}, \\
& \frac{\mathrm{d} n}{\mathrm{~d} t}=\frac{\mathrm{d} n_{\mathrm{I}}^{1}}{\mathrm{~d} t}+\frac{\mathrm{d} n_{\mathrm{II}}^{1}}{\mathrm{~d} t}=\left(\alpha v_{0}-v\right) \frac{\pi r_{\mathrm{s}}^{2}}{\Omega} .
\end{aligned}
$$

Since metal atoms (M) are supposed to neither evaporate nor precipitate, there exists the relation that $\frac{n}{n_{\mathrm{M}}}=\frac{c}{1-c}$, which can be further expressed as

$$
c=\frac{n}{n_{\mathrm{M}}+n}=\frac{n}{\frac{2 / 3 \pi r_{1, \mathrm{o}}^{3}\left(1-c_{0}\right)}{\Omega}+n},
$$

where $r_{l, \mathrm{o}}$ is the droplet radius at the onset of II. Since $r_{\mathrm{s}}$ is constant and $c$ is completely determined by $n, v$ becomes a function of only $n$. The nanowire growth rate $v$ is an explicit function of $c$ and $r_{\mathrm{s}}$ [10], we will denote it as $v(n)$. Now (15) becomes

$$
\frac{\mathrm{d} n}{\mathrm{~d} t}=\left(\alpha v_{0}-v(n)\right) \frac{\pi r_{\mathrm{s}}^{2}}{\Omega},
$$




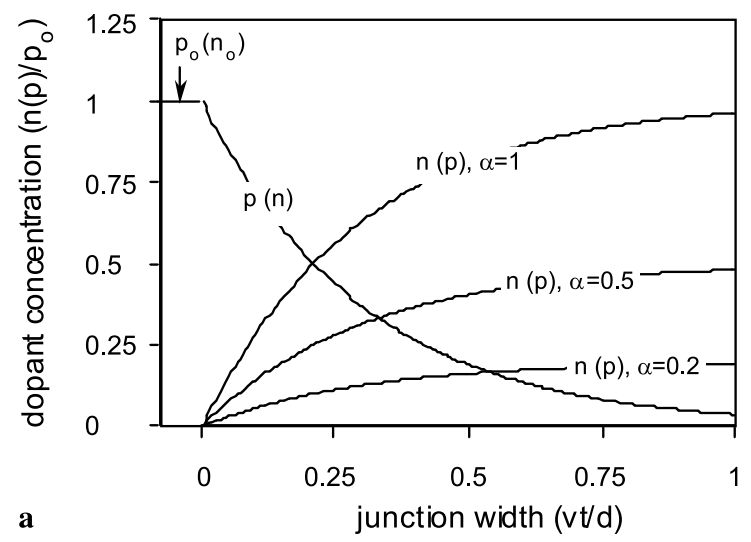

FIGURE 6 Normalized concentration profiles for pn-junctions under different second dopant (II) strengths, all of which are smaller than the first dopant (I) strength: (a) The dopant concentration profiles, and (b) The carrier concentration profile

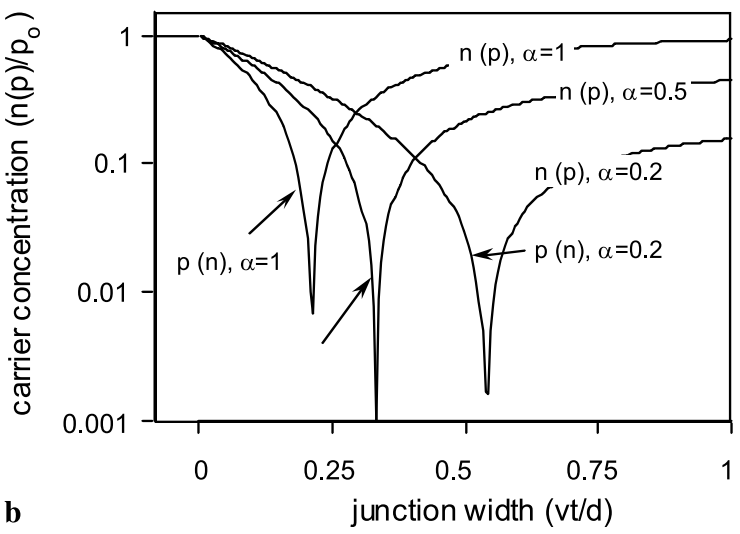

which is an ordinary differential equation of $n$. Solve (17) numerically with $n=n_{0}$ at $t=0$, and thus we obtain $n$ and $v$ for every instant $t$. Then using (13) and (14), we can also obtain $n_{1}$ and $n_{2}$ at $t$. Finally with $c_{\mathrm{I} / \mathrm{II}}^{\mathrm{s}}=\frac{n_{\mathrm{I} / \mathrm{II}}}{n}$ and $L_{\mathrm{j}}=$ $\int v \mathrm{~d} t$, we can describe the concentration variations along the heterojunction transition region.

Although we can study the relevant heterojunction features using simulation based on the above method, there are difficulties in accurately analyzing experimental profiles. First, values for some of the involved parameters can only be estimated. Second, I and II can behave differently both in the dissociation reaction of precursors and on the segregation effect at the liquid-solid interface. This can sometimes explain the large difference of abruptness between the leading edge and the trailing edge of II and needs to be taken into account in future studies, both theoretically and experimentally.

\section{Conclusions}

In conclusion, we mention that a model has been proposed to describe the graded composition transition of the heterojunctions and pn-junctions in nanowires grown by the VLS method. Our model yields satisfactory fit to a set of available experimental results. According to our model, it is in principle impossible to grow a heterojunction with a junction width on the monolayer scale via the VLS method. The abruptness of the heterojunction depends on the semiconductor solubility in the liquid metal droplet, which explains why for some III-V compounds, sharper heterojunctions can be obtained than for Si-Ge heterojunctions with broader widths that are a sizable fraction of the wire diameter. The junction width of a pn-junction can be adjusted by dopant strengths. The growth sequence of using a smaller dopant concentration followed by a higher dopant concentration of the opposite type yields shaper pn-junctions. Since the junction width is directly proportional to the wire diameter the problem of junction width on an absolute length scale becomes alleviated for nanowires in the diameter range of a few nanometers, which is the range in which nanowires might be applied for future nanoelectronic devices.

ACKNOWLEDGEMENTS The authors acknowledge the courtesy of Drs. T.E. Clark, E.C. Dickey, K.K. Lew, P. Nimmatoori, L.P., and J.M. Redwing of Pennsylvania State University for allowing us to use their experimental data [5] that are yet to be formally published.

\section{REFERENCES}

1 S. Gudiksen, L. Lauhon, J. Wang, D. Smith, C. Lieber, Nature 415, 617 (2002)

2 T. Koga, X. Sun, S. Cronin, M. Dresselhaus, Appl. Phys. Lett. 75, 2438 (1999)

3 Y. Wu, R. Fan, P. Yang, Nano Lett. 2, 83 (2002)

4 N.D. Zakharov, P. Werner, G. Gerth, L. Schubert, L. Sokolov, U. Goesele, J. Cryst. Growth 290, 6 (2006)

5 T.E. Clark, E.C. Dickey, K.K. Lew, P. Nimmatoori, J.M. Redwing, paper DD18.5, "Diameter Dependence of Interfacial Abruptness in $\mathrm{Si} / \mathrm{Si}_{1-x} \mathrm{Ge}_{x}$ Axial Heterostructure Nanowires Grown via Vapor-Liquid-Solid Growth", Symposium DD: Low-Dimensional Materials-Synthesis, Assembly, Property Scaling, and Modeling, Materials Research Society 2007 Spring Meeting, San Francisco, April 9-13 (2007)

6 M.T. Bjoerk, B.J. Ohlsson, T. Sass, A.I. Persson, C. Thelander, M.H. Magnusson, K. Deppert, L.R. Wallenberg, L. Samuelson, Nano Lett. 2, 87 (2002)

7 M.A. Verheijen, G. Immink, T. de Smet, M.T. Borgstroem, E.P.A.M. Bakkers, J. Am. Chem. Soc. 128, 1353 (2006)

8 M.T. Borgstroem, M.A. Verheijen, G. Immink, T. de Smet, E.P.A.M. Bakkers, Nanotechnology 17, 4010 (2006)

9 M.T. Bjoerk, C. Thelander, A.E. Hansen, L.E. Jensen, M.W. Larsson, L.R. Wallenberg, L. Samuelson, Nano Lett. 4, 1621 (2004)

10 L. Schubert, P. Werner, N.D. Zakharov, G. Gerth, F.M. Kolb, L. Long, U. Gösele, T.Y. Tan, Appl. Phys. Lett. 84, 4968 (2004) 